3 Research Square

\title{
An in-silico study of cartilage tissue engineering and its primary developmental growth factors in a systems biology approach
}

Nima Beheshtizadeh ( $\sim$ N-Beheshtizadeh@Razi.tums.ac.ir)

\section{Research}

Keywords: In-silico study, PPI network, cartilage tissue engineering, systems biology, regenerative medicine

Posted Date: July 2nd, 2020

DOI: https://doi.org/10.21203/rs.3.rs-38853/v1

License: (c) (1) This work is licensed under a Creative Commons Attribution 4.0 International License.

Read Full License 


\section{Abstract \\ Background}

Numerous growth factors work in consonance to regulate the development and homeostasis of engineered cartilage. In order to fabricate mature cartilages provided to implantation, developing biological processes should be known and employed. In this regard, a systems biology study could be considered as an inexpensive evaluation method. In this work, a systems biology study was performed on 11 protein-coding genes participating in cartilage development.

\section{Results}

Developing a protein-protein interaction (PPI) network resulted in a graph with 11 nodes and 28 edges. Additionally, a gene ontology (GO) analysis and a centrality analysis were performed based on the degree index. Four most crucial biological processes involved in cartilage engineering identified by the gene ontology analysis: cartilage development, cartilage condensation, regulation of cartilage development, and cartilage development involved in endochondral bone morphogenesis. Also, seven most interactive proteins were detected performing a degree-based centrality analysis: COL2A1, SOX9, CTGF, BMP7, TGFB1, CD44, and MAPK14.

\section{Conclusion}

Cartilage developmental proteins are efficient in forming and developing extracellular matrix and cartilage development, from its formation to the mature structure. However, this in-silico study recommends four most important developmental growth factors applicable to cartilage tissue engineering: COL2A1, SOX9, CTGF, and TGFB1.

\section{Introduction}

Tissue engineering combines the principles of cells, growth factors, and biomaterials to develop substitute tissues and promote endogenous regeneration [1]. Tissue engineering, as well as cell and gene therapy, could be considered as regenerative medicine, which is now attainable as a novel curing strategy [2]. Fabricating body tissues such as cornea or cartilage and organs like skin and bone by tissue engineering strategies would lead to shortening the transplant waiting list, which is extending every 15 minutes by adding a patient name [3].

Cartilage is a rubbery, stiff, and connective tissue that depicts a limited potential of self-regeneration due to the sparse cellularity, diminished production of the extracellular matrix (ECM), and lack of blood vessels and neural innervations $[4,5]$. Cartilage tissue engineering is one of the most appealing areas among scientists and clinicians, as the demand for cartilage repair and replacement increases 
considerably. On the one hand, cartilage diseases grow, so does the need for engineered cartilage tissues. Also, the progress of a sedentary lifestyle causes more bone and cartilage disorders. On the other hand, current surgical treatments for cartilage injuries are not satisfactory. Therefore, there is a climbing demand for novel strategies to induce cartilage development [6]. By introducing biocompatible polymers and hydrogels, combined with cells and growth factors for cartilage repair, regenerative medicine supplies an alternative solution [7]. Hence, it is predictable that cartilage tissue engineering's upward trend would be amplified in the foreseeable future [8].

There has been a dramatic progression in cartilage engineering during recent years [9]. Studies show that tissue engineering and regenerative medicine facilities were used to overcome cartilage diseases [9-11]. Atoufi et al. [12] produced an injectable thermosensitive Poly (N-isopropyl-acrylamide) /hyaluronic acid hydrogels containing various amounts of Chitosan-g-acrylic acid-coated poly (lactic-co-glycolic acid) (PLGA) micro and nanoparticles to facilitate the development of cartilage tissue. They claimed that due to the desired mechanical properties, high bioactivity, and sustainable drug release ability, the mentioned injectable hydrogel is a promising biomaterial for cartilage tissue engineering. Chen et al. [13] developed a porous scaffold prepared by gelatin groove fibers. They could obtain a considerable water absorption property of scaffolds as high as $1187 \%$. In their study, scaffolds compressive mechanical property was also evaluated. Upon their results, the scaffold above demonstrated elastic behavior in wet mode and could bear 100 cycles compressive fatigue test. Moreover, the test of this type of cartilage scaffold was successful in promoting rat chondrocyte and bone marrow mesenchymal stem cells (BMSC) proliferation.

Meanwhile, Al-Sabah et al. [14] developed a composite of natural biopolymer derived from cellulose, which is Nano-cellulose, and sodium alginate. They aimed to obtain a novel ink for cartilage scaffold 3D printing. They studied the impact of various calcium chloride cross-linker concentrations and sterilization strategies on the structural and mechanical properties of Nano-cellulose-based hydrogels for cartilage scaffolds. These scaffolds were containing plant-derived cellulose Nano-fibrils, cellulose nanocrystals, or a blend of the two.

In addition to the biomaterial determination for scaffold preparation, accurately selecting of various available cells are vital $[15,16]$. Chen et al. [17] studied the effect of utilizing diverse bone marrow-derived cell sources, including bone marrow concentrate (BMC) and BMSC for cartilage tissue engineering. The biological constructs containing BMC/BMSCs and articular tissue fragments were examined in vitro. Their results illustrated the biological constructs containing BMC and articular fragments contributed to immeasurable chondrogenesis. Therefore, it can be thought that BMC would be a potential candidate for a cell source for cartilage tissue engineering. Moreover, scientists studied the immune reaction and the degradation of the scaffold's fate in the cartilage engineering field [18-21].

Growth factors, a group of biologically active polypeptides produced by the body, could stimulate cellular division, growth, and differentiation [22]. In articular cartilage, numerous growth factors work in consonance to regulate the development and homeostasis of articular cartilage throughout life [23]. They 
offer promising treatments for enhanced development of cartilage in focal articular cartilage defects or in situations of more widespread cartilage loss, such as those that occur in osteoarthritis (OA) $[24,25]$.

Considering growth factors and their roles in signaling pathways, the progressive trend of each protein's role determination in cartilage development, is remarkable. Fortier et al. [26] studied the role of growth factors in cartilage repair. They gathered all the known proteins in their study that were involved in this process. Mueller et al. [27] studied the regulatory mechanisms for cartilage and tendon cell phenotypes. In their study, known master regulators are predicted to be associated with the observed phenotypes, including TGF- $\beta$ in monolayer cultures and PDGF BB in 3D cultures.

Considering the independent up-ward trend of tissue engineering, and systems biology, a remarkable synergy could be available in consonance with computational science (Fig. 1). Drawing a network of relationships among potential growth factors to select them accurately could be valuable in this order. Based on published works, it can be concluded that there is no comprehensive systems biology study on the cartilage growth factors that can draw up the existing relationship between them. Besides, to the best of our knowledge, there is no in-silico study of cartilage tissue engineering. In fact, in cartilage formation, several proteins are used therapeutically [28]. However, there are so few resources that could demonstrate the most impressive of them to accelerate chondrocyte growth. Also, there is no available information about which protein plays a more significant role in the regulation of cartilage development.

[Insert Fig. 1]

Although accumulating the best growth factors based on the previous experiments and the existing data is accessible via in-vitro and in-vivo studies, there are many reports about the effects of using each growth factor without any coherence and correlation.

If there are some interactions between growth factors, the main issue is to find them out. Hence, in this work, we aim to define a network for considering the relation among cartilage growth factors.

\section{Materials And Methods}

A majority of in-vivo and in-vitro studies already performed on cartilage tissue engineering demonstrated environmental factors surrounding the cells. The final results of these studies were gathered in some databases to provide comprehensive and accurate information. Regeneration gene database is one of these useful and applicable databases [29], According to this database, 11 protein-coding genes are participating in cartilage development. To find out their interaction and realize how they affect each other, all of these proteins were collected from this database. Then a systems biology study was performed. The mathematical modeling of complicated biological systems is called systems biology [30]. For this purpose "STRING App" in "Cytoscape 3.7.2 software" was utilized. The STRING App is one of the Cytoscape software apps related to the String database [31]. This database is utilized for investigating the protein-protein interaction (PPI). 
PPI networks offer some biological and biomedical applications via determining significant molecular and cellular mechanisms [32]. Although some PPI detecting methods such as in vitro [33] and in vivo [34] techniques are available, in silico method is preferred yet, due to its ability in predicting interactions with $100 \%$ accuracy [35]. Identification of each protein's role in biological functions causes the better utilizing of their related interactions. Additionally, PPIs could serve as a starting point for further lab experiments [35].

Creating a PPI network, there are 11 nodes and 28 edges. On a lighter note, an 11 node-included graph was drawn by STRING App. Then, a gene ontology (GO) analysis was performed. GO analysis is a major Bioinformatics initiative to unify the representation of gene and gene product attributes across all species [36]. These analysis results are accounted for the Homo sapiens organism.

Additionally, a centrality analysis was performed based on the degree index. In network analysis, based on graph theory, centrality indicators identify the most important nodes within a network. The results of this analysis lead to a degree-based array of nodes in the network. In order to illustrate comprehensible figures, the circular layout was selected. Figure 2 shows this layout.

[Insert Fig. 2]

\section{Results}

Four most outstanding biological processes were studied in this work. "Cartilage development", "cartilage condensation", "regulation of cartilage development", and "cartilage development involved in endochondral bone morphogenesis" are the four most essential processes that impress the cartilage formation.

In tissue engineering approaches, the final goal is to fabricate a mature tissue in a laboratory to graft to the patient. The most reliable engineered tissue is the most similar one to the real tissue. Hence, using the most critical growth factors to differentiate stem cells and precursors accurately into the chondrocytes would be a vital fact. Also, it is noteworthy that just a few growth factors should be used in cell culture and tissue growing phases. Therefore, it is essential to note that which growth factors would lead to fabricating the most similar engineered cartilage to the real one. The protein-coding genes which are involved in cartilage development, are listed in Table 1. [Insert Table 1] 
Table 1

The list of protein-coding genes that are involved in cartilage development.

\begin{tabular}{|llll|}
\hline No. & Name & Gene ID & Degree \\
\hline 1 & SOX9 & 6662 & 9 \\
\hline 2 & MMP3 & 4314 & 7 \\
\hline 3 & COL2A1 & 1280 & 7 \\
\hline 4 & CD44 & 960 & 7 \\
\hline 5 & TGFB1 & 7040 & 6 \\
\hline 6 & CTGF & 1490 & 6 \\
\hline 7 & MAPK14 & 1432 & 4 \\
\hline 8 & BMP7 & 655 & 4 \\
\hline 9 & COL10A1 & 1300 & 3 \\
\hline 10 & S100B & 6285 & 2 \\
\hline 11 & S100A1 & 6271 & 1 \\
\hline
\end{tabular}

By performing a gene ontology analysis, the results would be accessible. As shown in Table 2, each protein is active in a biological process that leads to inevitable consequences. In this table, seven most interactive proteins among all 11 proteins were selected and discussed. These proteins are collagen type II alpha 1 chain (COL2A1), SRY-box 9 (SOX9), connective tissue growth factor (CTGF), bone morphogenetic protein 7 (BMP7), transforming growth factor-beta 1 (TGFB1), CD44, and mitogenactivated protein kinase 14 (MAPK14). In PPI networks, it is common to illustrate the relationship between proteins by hierarchical layout. In this layout, proteins connect in a line (Fig. 3).

Table 2

Proteins involved in cartilage developmental processes

\begin{tabular}{|llllllll|}
\hline $\begin{array}{l}\text { Biological Process/ Protein } \\
\text { Names }\end{array}$ & COL2A1 & SOX9 & CTGF & BMP7 & TGFB1 & CD44 & MAPK14 \\
\hline Cartilage Development & $*$ & $*$ & $*$ & $*$ & $*$ & $*$ & $*$ \\
\hline Cartilage Condensation & $*$ & $*$ & $*$ & & & & $*$ \\
\hline $\begin{array}{l}\text { Regulation of Cartilage } \\
\text { Development }\end{array}$ & & $*$ & $*$ & & $*$ & & \\
\hline $\begin{array}{l}\text { Cartilage Development } \\
\text { Involved in Endochondral } \\
\text { Bone Morphogenesis }\end{array}$ & $*$ & $*$ & & & & & \\
\hline
\end{tabular}

[Insert Table 2] 
[Insert Fig. 3]

\section{Discussion}

Based on GO analysis, the four most effective biological processes and their involved proteins are shown in Table 2. Cartilage development (GO: 0051216) defined as a process whose specific outcome is the progression of the cartilage element over time, from its formation to the mature structure [37]. A glance at the table provided reveals that all seven proteins mentioned above are active in this process. On a lighter note, each protein of these seven ones could be a vital growth factor in cartilage tissue engineering as a performer of the cartilage development process.

Moreover, based on GO terminology, condensation of the mesenchymal cells process that has been committed to differentiating into chondrocytes is known as cartilage condensation (GO: 0001502) [38]. COL2A1, SOX9, CTGF, and MAPK14 are four active proteins in this biological procedure. These proteins possess the ability to impress the mesenchymal cells as a growth factor to condensate and differentiate into chondrocytes. These proteins are shown in Fig. 4.

[Insert Fig. 4]

Furthermore, any process that modulates the rate, frequency, or extent of cartilage development could define as regulation of cartilage development (GO: 0061035) [39]. Based on this description, SOX9, CTGF, and TGFB1 are the primary growth factors involved in cartilage development regulation (Fig. 5). The process, whose specific outcome is the cartilage's progression, will provide a scaffold for mineralization of endochondral bones, known as cartilage development, involved in endochondral bone morphogenesis (GO: 0060351) [40]. SOX9 and COL2A1 are two active proteins in this trend. This biological process occurs in cartilage formation from infant to the youth and does not involve cartilage tissue engineering [40]. However, its role is so vital in endochondral bone morphogenesis. Figure 6 shows this procedure's active proteins.

[Insert Fig. 5]

[Insert Fig. 6]

Regarding the information extracted from charts and tables, these biological processes are irrefutable in cartilage formation. Although some other well-known growth factors are playing a significant role in the chondrocyte differentiation [41, 42], like Platelet-derived growth factor (PDGF) [43], insulin-like growth factor I (IGF-I) [44], and Fibroblast growth factor (FGF) [45], the progressive trend of the cartilage development, from its formation to the mature structure needs some cartilage developmental growth factors that are summarized in Table 2. The formation and development of ECM containing collagen type II and large amounts of proteoglycan, mainly chondroitin sulfate, demand the mentioned growth factors to pass biological processes. 


\section{Conclusion}

As a corollary, in articular cartilage, numerous growth factors work in concert to regulate the development and homeostasis of articular cartilage. In order to fabricate mature cartilages provided to implantation, developing biological processes should be known and employed. Some growth factors like PDGF, IGF-I, and FGF, affect chondrocyte differentiation; whereas, cartilage developmental growth factors are so beneficial in chondrocyte differentiation as well as the formation and development of extracellular matrix, leading to cartilage evolution, from its formation to the mature structure. Notwithstanding, based on limitations on growth factors employment, four most prominent developmental growth factors are recommended for cartilage tissue engineering as COL2A1, SOX9, CTGF, and TGFB1.

\section{Abbreviations}

Extracellular matrix (ECM)

Poly (lactic-co-glycolic acid) (PLGA)

Bone marrow mesenchymal stem cells (BMSC)

Bone marrow concentrate (BMC)

Osteoarthritis (OA)

Protein-protein interaction (PPI)

Gene ontology (GO)

Collagen type II alpha 1 chain (COL2A1)

SRY-box 9 (SOX9)

Connective tissue growth factor (CTGF)

Bone morphogenetic protein 7 (BMP7)

Transforming growth factor beta 1 (TGFB1)

Mitogen-activated protein kinase 14 (MAPK14)

Platelet-derived growth factor (PDGF)

Insulin-like growth factor I (IGF-I)

Fibroblast growth factor (FGF) 


\section{Declarations}

Acknowledgments: Not applicable.

Funding: This study was not funded by any funding institute.

Availability of data and materials: The data sets used and/or analyzed during the current study are available from the corresponding author on reasonable request.

Ethics approval and consent to participate: Not applicable.

Consent for publication: Not applicable.

Competing interests: The authors declare that they have no competing interests

Ethical approval: This article does not contain any studies with human participants or animals performed by any of the authors.

\section{References}

1. Samadikuchaksaraei A, et al., Chap. 4 - Stem Cells as Building Blocks, in Principles of Tissue Engineering (Fourth Edition), R. Lanza, R. Langer, and J. Vacanti, Editors. 2014, Academic Press: Boston. p. 41-55.

2. Dzobo K, et al., Advances in Regenerative Medicine and Tissue Engineering: Innovation and Transformation of Medicine. Stem cells international, 2018. 2018: p. 2495848-2495848.

3. Hsich EM. Matching the Market for Heart Transplantation. Circ Heart Fail. 2016;9(4):e002679-9.

4. Claire V, et al. "Cartilage Tissue Engineering: Towards a Biomaterial-Assisted Mesenchymal Stem Cell Therapy". Curr Stem Cell Res Ther. 2009;4(4):318-29.

5. Sá-Lima $\mathrm{H}$, et al. "Thermoresponsive poly(N-isopropylacrylamide)-g-methylcellulose hydrogel as a three-dimensional extracellular matrix for cartilage-engineered applications". Journal of Biomedical Materials Research Part A. 2011;98A(4):596-603.

6. Mendelson A, et al. "Engineered nasal cartilage by cell homing: A model for augmentative and reconstructive rhinoplasty". Plast Reconstr Surg. 2014;133(6):1344-53.

7. Khoshfetrat AB, et al. "Enzymatically-gellable galactosylated chitosan: Hydrogel characteristics and hepatic cell behavior". Int J Biol Macromol. 2016;92:892-9.

8. Chen F-M, Liu X. Advancing biomaterials of human origin for tissue engineering. Prog Polym Sci. 2016;53:86-168.

9. Ringe J, Sittinger M. "Tissue engineering in the rheumatic diseases". Arthritis research therapy. 2009;11(1):211-1.

10. Jorgensen $C$, et al. "Stem cells for repair of cartilage and bone: the next challenge in osteoarthritis and rheumatoid arthritis". Ann Rheum Dis. 2001;60(4):305-9. 
11. Martín AR, et al, "Emerging therapies for cartilage regeneration in currently excluded 'red. knee' populations". npj Regenerative Medicine, 2019. 4(1): p. 12.

12. Atoufi Z, et al. "Injectable PNIPAM/Hyaluronic acid hydrogels containing multipurpose modified particles for cartilage tissue engineering: Synthesis, characterization, drug release and cell culture study". Int J Biol Macromol. 2019;139:1168-81.

13. Chen W, et al. "Groove fibers based porous scaffold for cartilage tissue engineering application". Mater Lett. 2017;192:44-7.

14. Al-Sabah A, et al. "Structural and mechanical characterization of crosslinked and sterilised nanocellulose-based hydrogels for cartilage tissue engineering". Carbohyd Polym. 2019;212:242-51.

15. Hardingham T, Tew S, Murdoch A. "Tissue engineering: chondrocytes and cartilage". Arthritis Research Therapy. 2002;4(3):S63.

16. Tuli R, Li W-J, Tuan RS. "Current state of cartilage tissue engineering" Arthritis Res Ther. 2003;5(5):235.

17. Chen $\mathrm{C}-\mathrm{C}$, et al. "A comparison of distinct bone marrow-derived cells on cartilage tissue engineering". J Taiwan Inst Chem Eng. 2017;78:32-8.

18. Badylak SF, Gilbert TW. "Immune response to biologic scaffold materials". Seminars in immunology. 2008;20(2):109-16.

19. Li H, et al., "Immunomodulatory Functions of Mesenchymal Stem Cells in Tissue Engineering". Stem Cells International, 2019. 2019: p. 18.

20. Longoni $A$, et al. "The impact of immune response on endochondral bone regeneration". npj Regenerative Medicine. 2018;3(1):22.

21. Yang $D$, et al. "The immune reaction and degradation fate of scaffold in cartilage/bone tissue engineering". Materials Science Engineering: C. 2019;104:109927.

22. Lee K, Silva EA, Mooney DJ. "Growth factor delivery-based tissue engineering: general approaches and a review of recent developments". J R Soc Interface. 2011;8(55):153-70.

23. Goldring MB, Tsuchimochi K, ljiri K. "The control of chondrogenesis". J Cell Biochem. 2006;97(1):3344.

24. Civinini R, et al., "Growth factors in the treatment of early osteoarthritis". Clinical cases in mineral and bone metabolism: the official journal of the Italian Society of Osteoporosis, Mineral Metabolism, and Skeletal Diseases, 2013. 10(1): p. 26-29.

25. Huang J, Zhao L, Chen D. "Growth factor signalling in osteoarthritis" Growth Factors. 2018;36(56):187-95.

26. Fortier LA, et al. "The role of growth factors in cartilage repair". Clin Orthop Relat Res. 2011;469(10):2706-15.

27. Mueller AJ, et al. A systems biology approach to defining regulatory mechanisms for cartilage and tendon cell phenotypes. Sci Rep. 2016;6(1):33956. 
28. Steinert AF, Nöth U, Tuan RS. Concepts in gene therapy for cartilage repair. Injury. 2008;39(Suppl 1):S97-113. Suppl 1 ).

29. Zhao M, Wang BR,T, Cummins SF. "REGene: an online gene resource for animal regeneration with literature evidence"Sci Rep. 2016;6:srep25400.

30. Fischer HP. Mathematical modeling of complex biological systems: from parts lists to understanding systems behavior. Alcohol research health: the journal of the National Institute on Alcohol Abuse Alcoholism. 2008;31(1):49-59.

31. Doncheva NT, et al., "Cytoscape stringApp: Network analysis and visualization of proteomics data". J Proteome Res, 2018.

32. Safari-Alighiarloo N, et al. Protein-protein interaction networks (PPI) and complex diseases. Gastroenterology hepatology from bed to bench. 2014;7(1):17-31.

33. Rigaut $\mathrm{G}$, et al. A generic protein purification method for protein complex characterization and proteome exploration. Nat Biotechnol. 1999;17(10):1030-2.

34. Uetz $\mathrm{P}$, et al. A comprehensive analysis of protein-protein interactions in Saccharomyces cerevisiae. Nature. 2000;403(6770):623-7.

35. Rao VS, et al., Protein-Protein Interaction Detection: Methods and Analysis. International Journal of Proteomics, 2014. 2014: p. 147648.

36. Consortium TGO. "The Gene Ontology project in 2008". Nucleic Acids Res. 2007;36(suppl_1):D440-4.

37. Sophia Fox AJ, Bedi A, Rodeo SA. The basic science of articular cartilage: structure, composition, and function. Sports health. 2009;1(6):461-8.

38. Gadjanski I, Spiller K, Vunjak-Novakovic G. Time-dependent processes in stem cell-based tissue engineering of articular cartilage. Stem cell reviews reports. 2012;8(3):863-81.

39. Akkiraju $\mathrm{H}$, Nohe A. Role of Chondrocytes in Cartilage Formation, Progression of Osteoarthritis and Cartilage Regeneration. Journal of developmental biology. 2015;3(4):177-92.

40. Ortega N, Behonick DJ, Werb Z. Matrix remodeling during endochondral ossification. Trends in cell biology. 2004;14(2):86-93.

41. Cugat R, et al. "Biologic Enhancement of Cartilage Repair: The Role of Platelet-Rich Plasma and Other Commercially Available Growth Factors". Arthroscopy: The Journal of Arthroscopic Related Surgery. 2015;31(4):777-83.

42. Fortier LA, et al. The role of growth factors in cartilage repair. Clin Orthop Relat Res. 2011;469(10):2706-15.

43. Barbero A, et al. "Age related changes in human articular chondrocyte yield, proliferation and postexpansion chondrogenic capacity". Osteoarthritis Cartilage. 2004;12(6):476-84.

44. Madry $\mathrm{H}$, et al. "Cartilage constructs engineered from chondrocytes overexpressing IGF-I improve the repair of osteochondral defects in a rabbit model". European cells materials. 2013;25:229-47.

45. Green JD, et al. "Multifaceted signaling regulators of chondrogenesis: Implications in cartilage regeneration and tissue engineering". Genes diseases. 2015;2(4):307-27. 


\section{Figures}

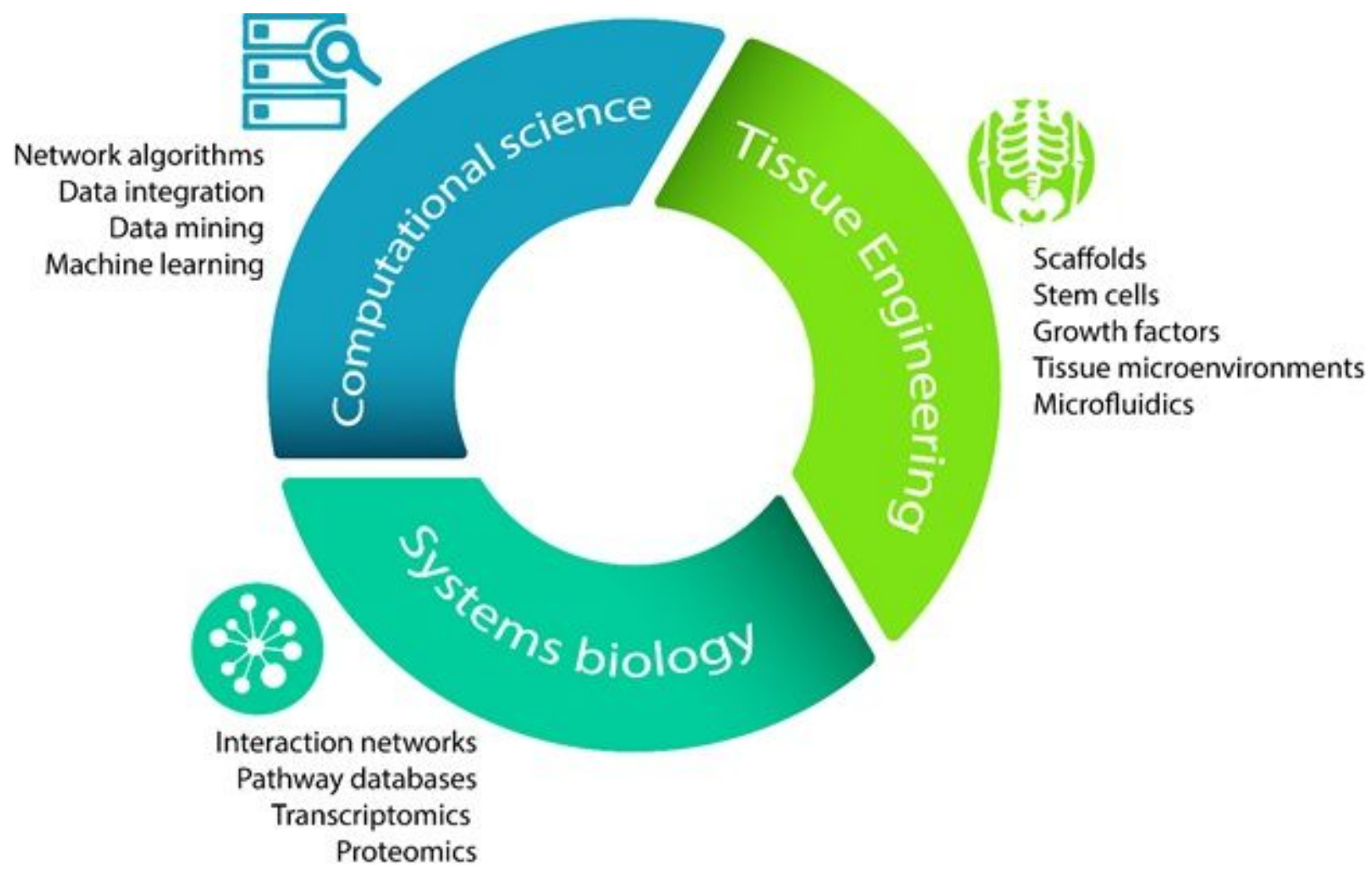

\section{Figure 1}

Science converging in the regenerative medicine field includes tissue engineering, systems biology, and computational science, which can influence and be mutually beneficial to one another. 


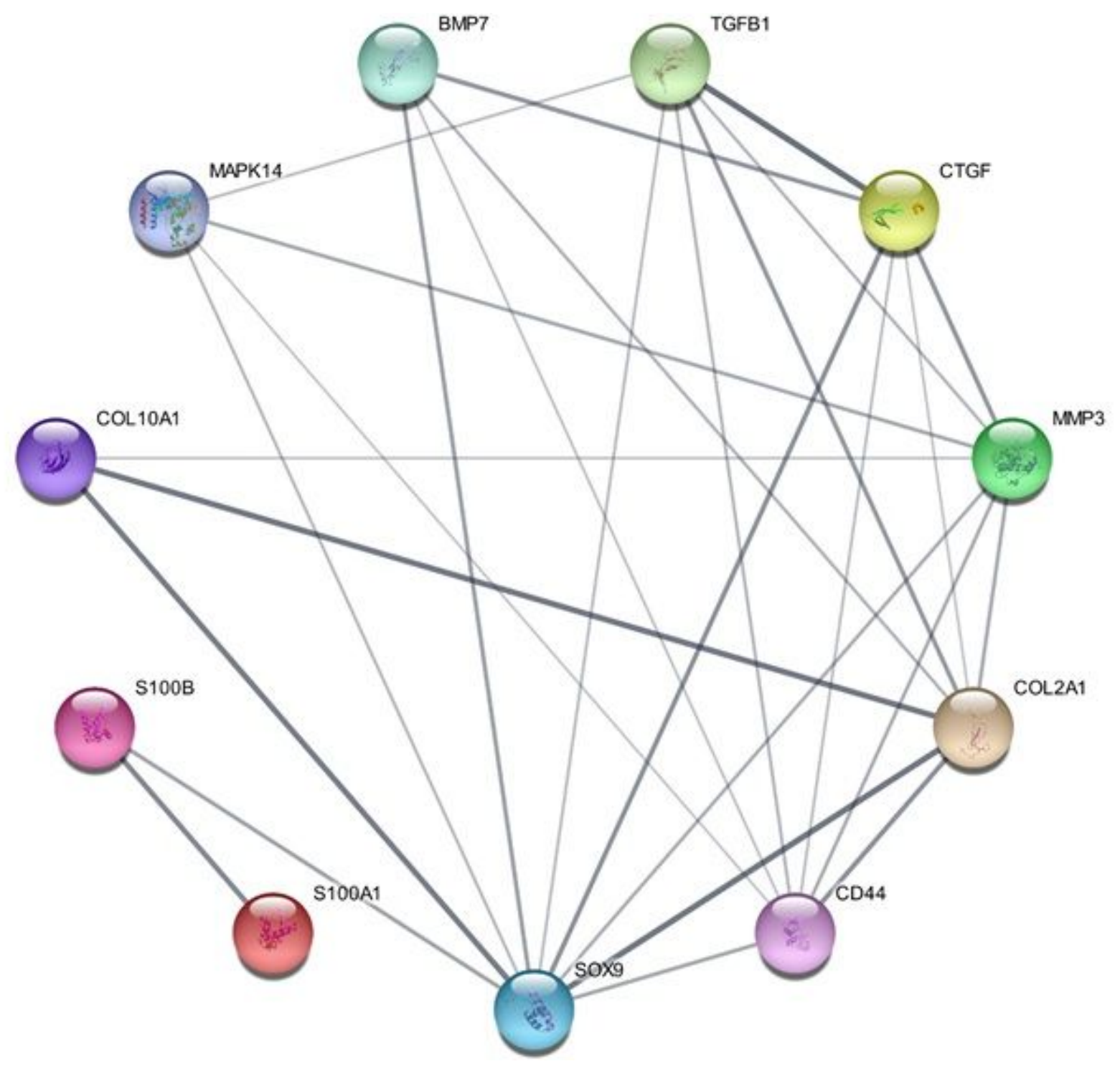

Figure 2

A degree-sorted circular layout of PPI network illustrating cartilage developmental growth factors. Each circle provides a schematic drawn of protein structure. The colors are set randomly, and the connection line's thickness illustrates the relation of power. Also, a thicker line presents much more evidence and documents to approve the connectivity. 


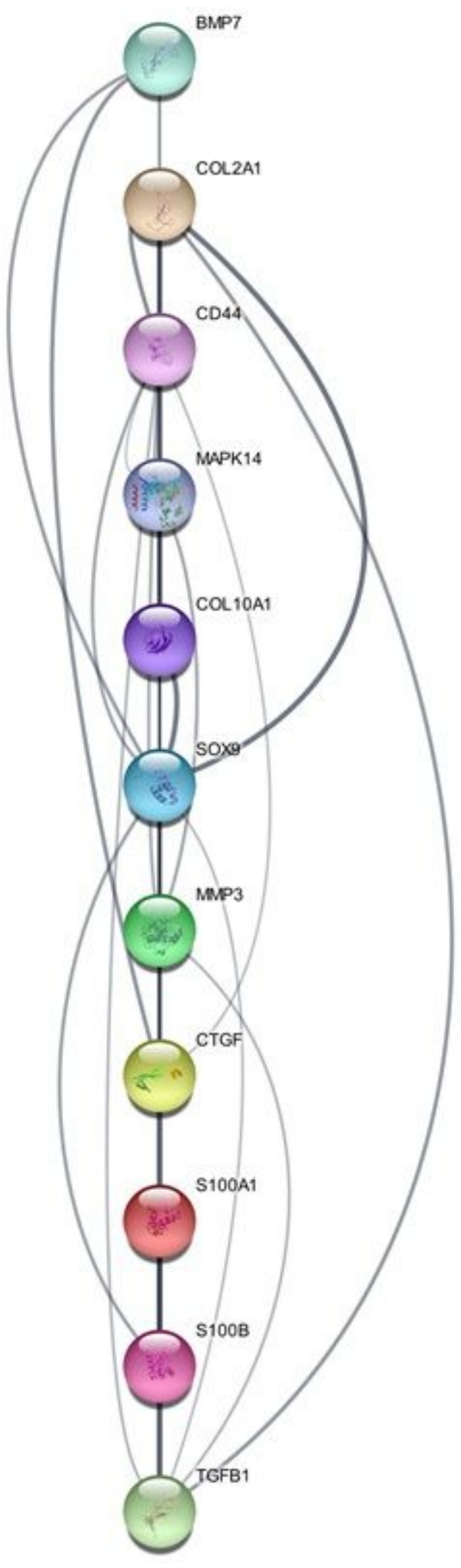

Figure 3

A Hierarchical layout of PPI network illustrating cartilage developmental growth factors. 


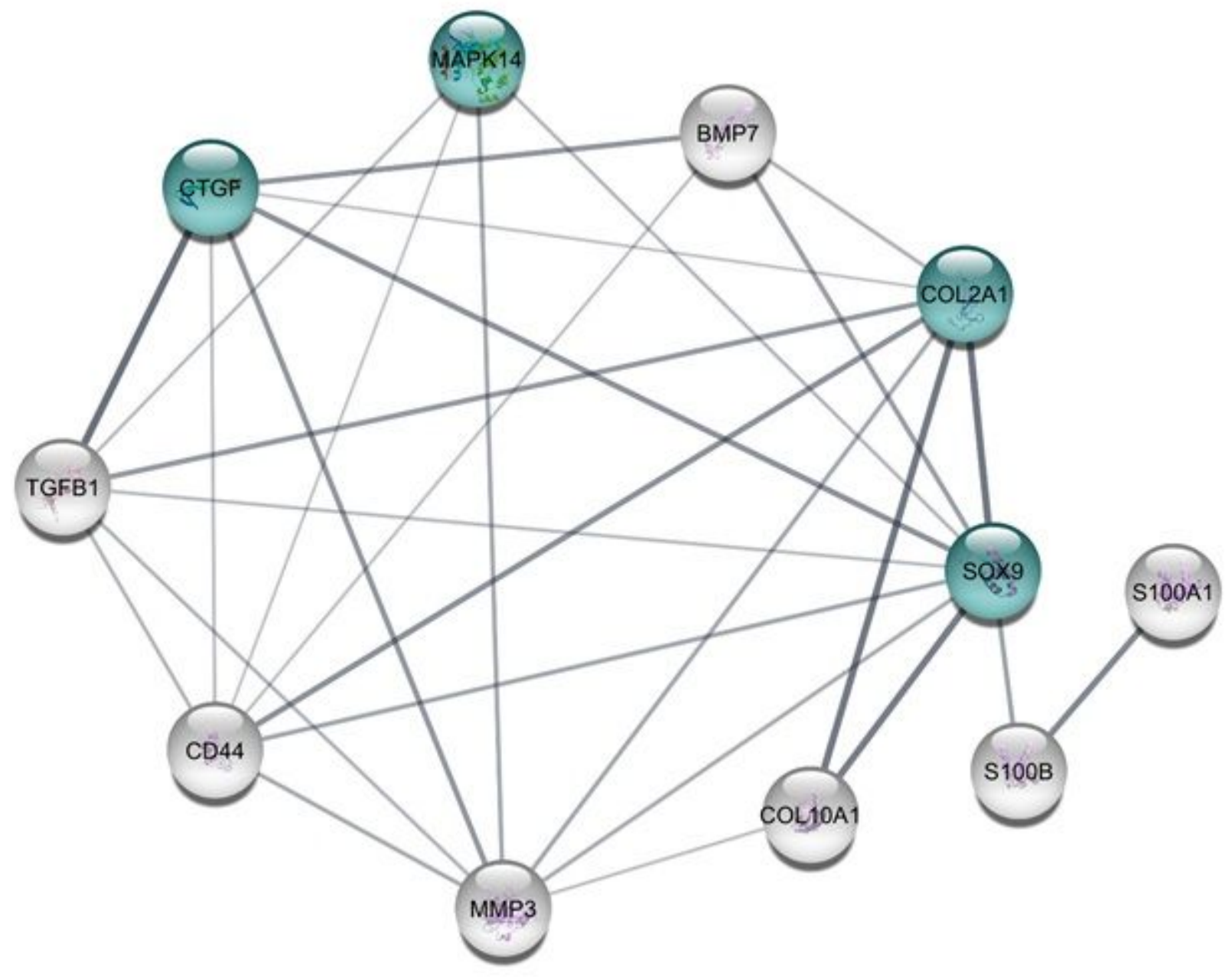

Figure 4

“Cartilage condensation” process involved proteins. 


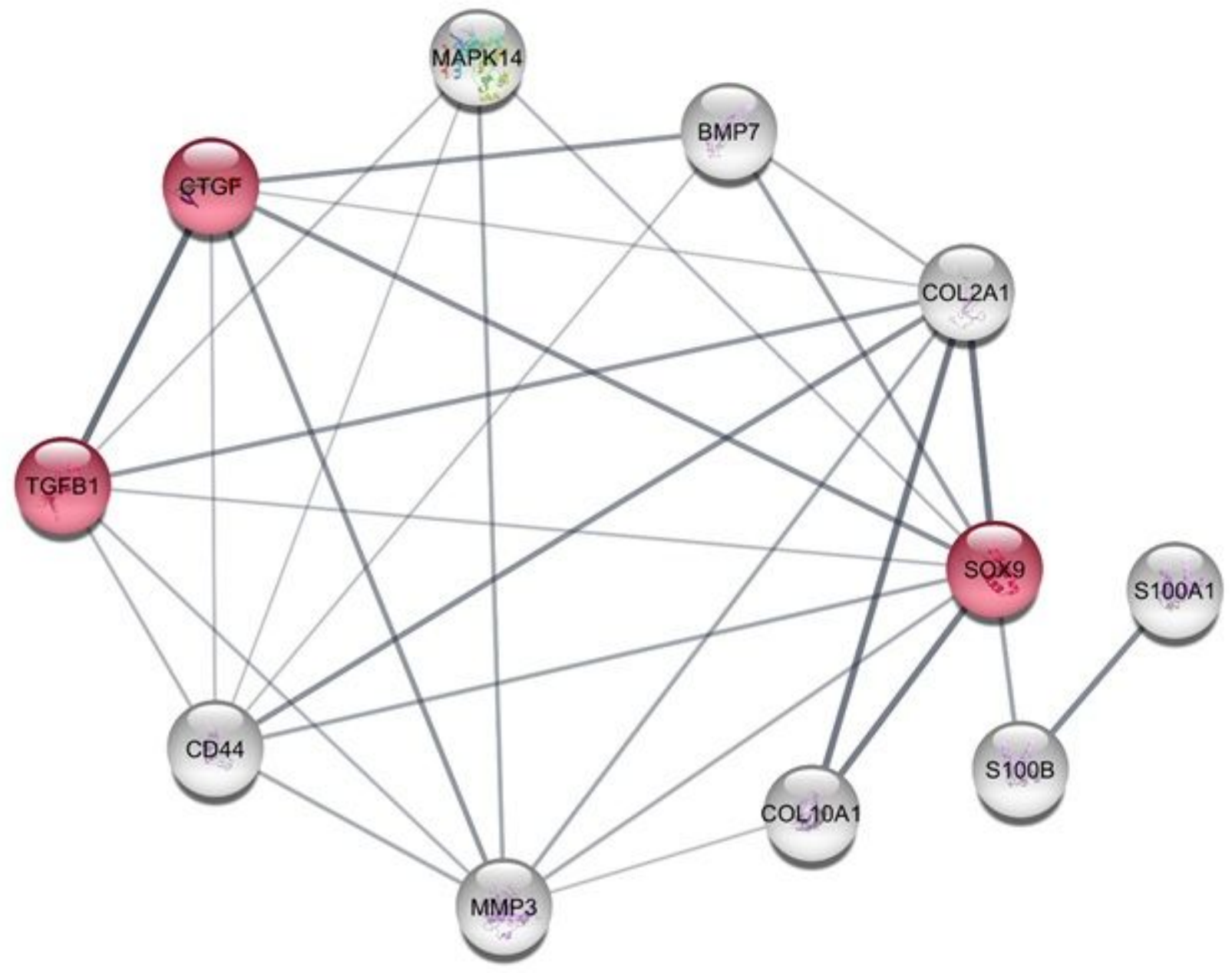

Figure 5

"Regulation of cartilage development" process involved proteins. 


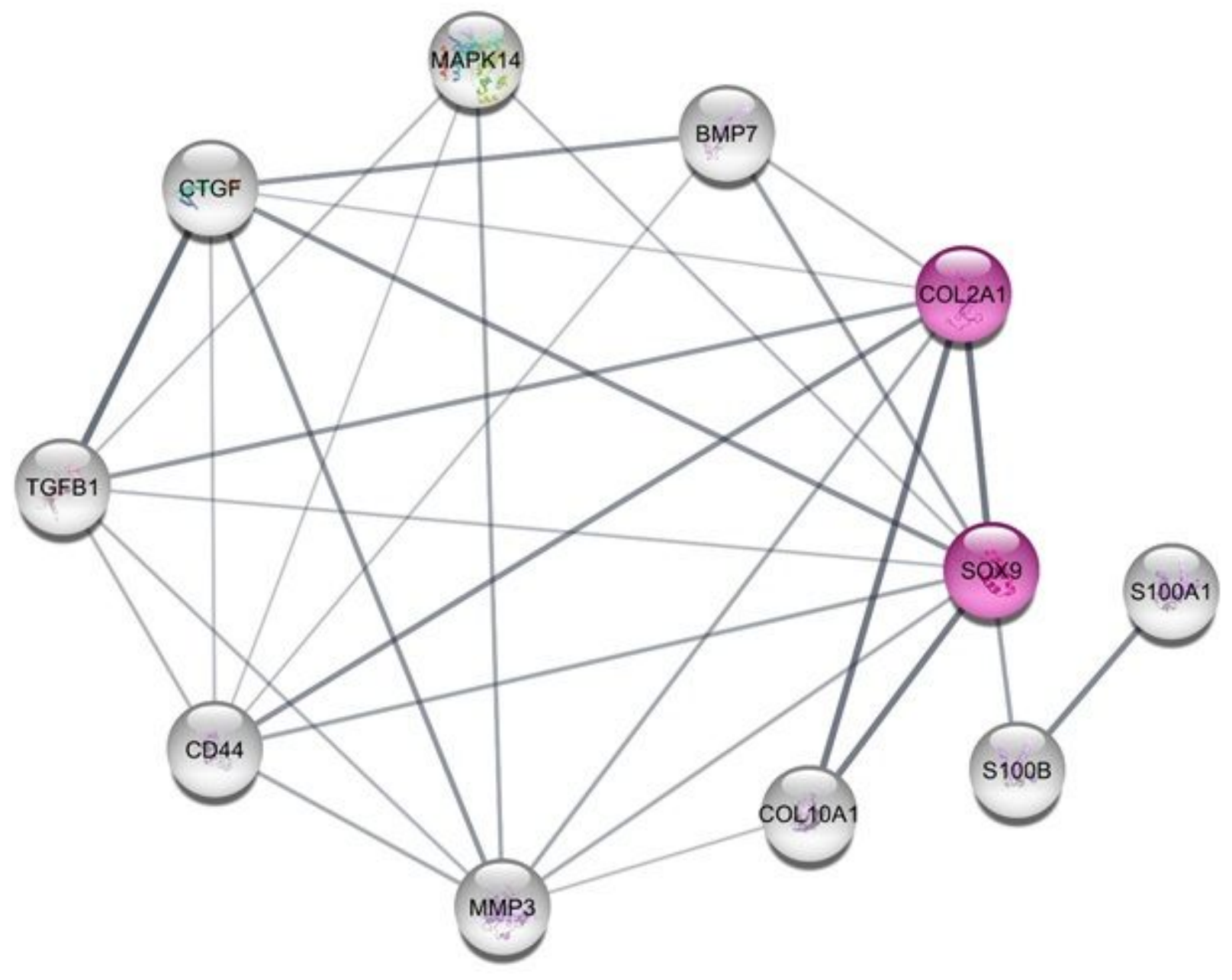

Figure 6

"Cartilage development involved in endochondral bone morphogenesis" process involved proteins. 